\title{
Application of Time-Frequency Direction Spectrum Analysis in Marine Electromagnetic Data Processing
}

\author{
Delong Tang', Chunhui Li ${ }^{1}$, Peng Lian ${ }^{2}$ \\ ${ }^{1}$ DaLian Ocean University, Dalian, Liaoning 116023, China \\ ${ }^{2}$ College of Fisheries, Ocean University of China, Qingdao, Shandong 266003, China
}

Keywords: Marine electromagnetism; Marine controllable source electromagnetic detection: Timefrequency directional spectrum; Application

\begin{abstract}
The electromagnetic environment in the ocean is opposite to that on land. It is an inverted environment. In the ocean, seawater has a high conductivity and covers the relatively high resistivity seabed strata. For quite a long time, marine gravity, marine magnetism, aeromagnetism, marine seismic survey and other methods were mostly used in marine survey and investigation fields, and electromagnetic detection methods were basically not used. In this paper, a set of marine controllable source electromagnetic data processing method is proposed, and the time-frequency and phase characteristics of marine environmental noise are analyzed through time-frequency directional spectrum. Decomposing the marine environmental noise into incoherent isotropic components and coherent anisotropic components can separate the contributions of various sources in the environmental noise to the actual sound field. The method of Fourier transform modulus maxima is used to denoise the electromagnetic signals from marine controllable sources. The obtained figure shows that the method has good denoising effect.
\end{abstract}

\section{Introduction}

With the increasing global energy demand and the decreasing reserves of oil and gas resources in onshore and shallow sea areas, deep sea oil and gas resources have become the focus of international energy competition, and offshore oil and gas exploration has also entered a climax. After decades of research and development, marine controlled source electromagnetic (CSEM) has matured in terms of hardware instruments, data processing, and data interpretation [1]. Conventional frequency domain marine electromagnetic exploration methods generally use horizontal dipole sources to emit low frequency electromagnetic waves to the seabed in deep sea areas. As an important method of marine geophysics, marine electromagnetic exploration has attracted more and more attention. The research on marine electromagnetic field theory theoretically guarantees the smooth development of marine electromagnetic exploration [2]. It is fully proved that the marine electromagnetic method can be applied to the marine environment, and also greatly improves the resolution of high resistivity thin oil and gas layers, which can not only fully make up for some deficiencies in seismic exploration, but also directly indicate oil and gas. This paper mainly puts forward the pretreatment method and flow of marine controllable source electromagnetic data, and illustrates the application effect of various treatment methods in detail with examples.

\section{Electromagnetic Signal from Ocean Controllable Source}

\subsection{Ocean CSEM sending signals}

Because of the strong conductivity of seawater, people usually regard the ocean as a low-pass filter. When we send a high-frequency electromagnetic signal to the seabed, the signal is seriously attenuated in the seawater. There is a global natural alternating electromagnetic field distributed over the earth. Its frequency range is very wide. Electromagnetic waves of different frequencies are superimposed on each other to form a very complex electromagnetic vibration. The electromagnetic 
field actually consists of two parts, one is transmitted in the surface layer and refracted to the underground, the other is transmitted directly from the ground and decays exponentially. In the case of isotropic noise field, the sound pressure and vibration velocity of noise are not correlated, and the target signal and environmental noise are also not correlated [3]. The air waves arrives almost at the same time, while the propagation speed of the guided wave in the high-resistance reservoir is much lower than the speed of light, which results in different time for the guided wave in the air waves and the high-resistance reservoir to reach the receiver at the same position. Low-frequency electromagnetic signals are commonly used in marine controllable source electromagnetic exploration, and low-frequency electromagnetic signals with a frequency in the range of $0.01 \mathrm{~Hz}$ to $100 \mathrm{~Hz}$ are emitted into seawater through a horizontal electric dipole source [4].

\subsection{Influence of emission frequency on ocean CSEM}

The physical properties of rocks in marine environment are different from those on land. Due to its high porosity and permeability, all the oceanic crust is actually saturated with seawater, especially the upper part of the oceanic crust. In the transition region between the near field region and the far field region, both the electric field and the magnetic field slowly transition from the near field region value to the far field region value. However, in heterogeneous media, the characteristics of the transition region are very complex [5]. However, they are generally periodic pulse sequences, such as square wave, trapezoidal wave, triangular wave, pseudo-random wave, etc. Different measurement conditions will cause changes in these characteristic physical quantities. When analyzing the characteristics of marine environmental noise, different measurement environments correspond to different characteristics of marine environmental noise vector fields. As the water depth decreases, the air waves component in the frequency domain electric field response dominates, flooding the effective signals from the seabed strata, causing the amplitude of the normalized electric field response to decrease [6]. Because the seabed electromagnetic acquisition station is placed on the seabed in a free sinking mode, the coordinate orientation of the acquisition station measurement system at the seabed is uncertain. The higher the incident frequency, the smaller the offset distance corresponding to the maximum amplitude anomaly. However, as the offset increases gradually, the stronger the influence of air waves on the signal, the higher the resistance response of the reservoir will be gradually overshadowed.

\subsection{Noise of marine electromagnetic signals}

Marine environmental noise can be divided into isotropic noise field and anisotropic noise field. Marine environmental noise field is the sum of coherent anisotropic field and incoherent isotropic field [7]. On the surface of the ocean, various disturbances, gravitational waves, seabed uplift, seismic waves and other reasons lead to electromagnetic field signals generated by fluctuations in seabed velocity and pressure. It runs at its peak at any time, so square wave has the advantage of transferring the maximum energy to the surface. Secondly, in the frequency domain, the current amplitude of the square wave is proportional to the reciprocal of the frequency, so the amplitude will decrease with the increase of the frequency and increase with the decrease.

Conductivity of marine sediments mainly depends on porosity and pore fluid filled therein, and obeys Archie's law [8]:

$$
\sigma_{m}=\frac{\Phi^{m} S^{n} \sigma_{\omega}}{A}
$$

Where $0.5<A<2.5$ is a constant, $S$ is the ratio of pores filled with water, $n$ is the saturation index, $\Phi$ is the porosity, $\sigma_{\omega}$ is the conductivity of pore water, and $\mathrm{m}$ is a constant mainly depending on the geometry of the pore space, called the degree of adhesion. For seawater saturated sediments, where $S=1$, equation (1) is simplified as: 


$$
\sigma_{m}=\frac{\Phi^{m} \sigma_{\omega}}{A}
$$

Generally speaking, the conductivity of seabed sediments is more than 1 times lower than that of seawater. Porosity and permeability of ocean crust are closely related to the age of rocks, so conductivity is also related to age.

The obtained time-frequency maps have much better clustering, but there are serious crossinterference items, which cause great confusion to the analysis of the original signals. However, the use of low-frequency electromagnetic data is easy to produce equivalent effect, that is, when the transverse resistance (the product of resistivity and layer thickness) of high-resistance thin layer is equivalent, its electric field response curve is basically consistent and difficult to distinguish. The amplitude of the wave magnetic field is proportional to the frequency, wave height and velocity. Some scholars have theoretically calculated the magnetic field spectrum of ocean waves by studying the ocean wave spectrum in oceanography, which is a narrow band spectrum whose amplitude in the geomagnetic field can reach several nanometers.

\section{Data Processing of Marine Controllable Source Electromagnetic Signal}

The initial processing of marine controllable source data is mainly divided into three steps: time domain filtering, unit conversion and time window adjustment analysis. As a result, the MVO curve of measured electric field amplitude varying with offset distance is calculated. So far, most of the ocean data are processed in the frequency domain. It is necessary to convert the measured binary data into the format required by the ocean CSEM data preprocessing program, and conduct time series analysis to understand the working state of the instrument and the quality of the collected data. Seawater wave signals arrive at a speed of $10 \mathrm{~km} / \mathrm{s}$ on the seabed, $1 \mathrm{~s}$ on the seabed, $100 \mathrm{~km} / \mathrm{s}$ on the oil and gas layer and $0.3 \mathrm{~s}$ on the oil and gas layer.

Fig. 1 is a normalized response curve of frequency domain and time domain impulse response data when seawater depth is $200 \mathrm{~m}$ and $300 \mathrm{~m}$ respectively and reservoir depth is $1000 \mathrm{~m}$. It can be seen that the time domain data is less affected by air waves, and when the water depth decreases, the normalized response amplitude increases instead, which indicates that the time domain data has strong identification ability for shallow sea high resistivity thin oil and gas layers.

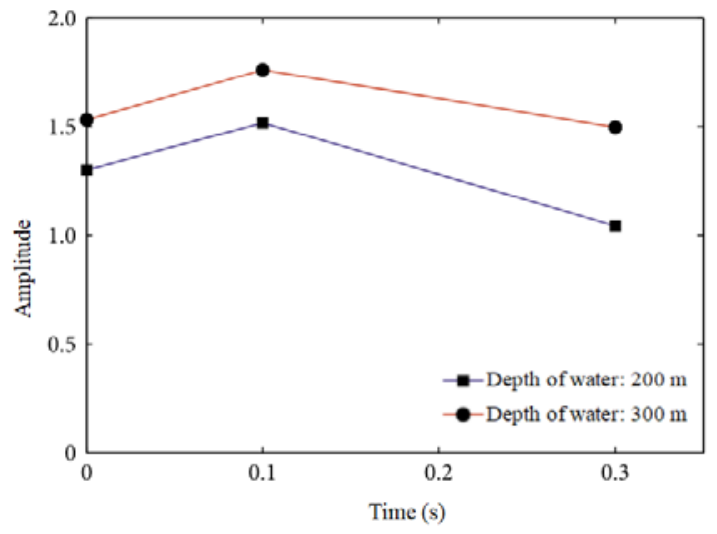

Figure 1 Normalized response curves for different water depths

The sensitivity of the velocity channel of the vector hydrophone is relatively low in the low frequency part. The sensitivity curve of the velocity channel rises slowly with respect to the frequency axis at frequencies below $50 \mathrm{~Hz}$. At frequencies above $50 \mathrm{~Hz}$, the slope of the sensitivity curve of the velocity channel with respect to the frequency axis meets $6 \mathrm{~d} B$ per octave. Because the low-frequency electromagnetic signals returned from the seabed medium are easily submerged in the "air waves" in shallow waters, the detected signals are difficult to identify and cannot be extracted. Azimuth correction processing mainly includes polarization ellipse analysis and rotating electrical axis. Using polarization ellipse analysis can obtain more accurate azimuth value, and 
rotating electrical axis can calculate the component of electric field in line direction.

In the marine electromagnetic construction, the seabed electromagnetic collection station is placed on the seabed in a free sinking way, and the position of the collection station is still unknown before the collection station is recovered. The physical meaning of phase spectrum is the distribution of phase difference between two quantities in frequency domain. When the phase spectrum of a component of sound pressure and vibration velocity is constant, it indicates that there is coherent interference in the marine environment in this direction, and the coherent anisotropic component in this direction accounts for the main component. fig. 2 is the normalized response curve of pulsed electric field in different time domains. As can be seen from the figure, when the offset distance is $0.6 \mathrm{~km}$ and $\mathrm{t}=1.5 \mathrm{~s}$, the normalized response value of time domain pulsed electric field is the largest, while the abnormal display of large offset distance and small offset distance is not obvious.

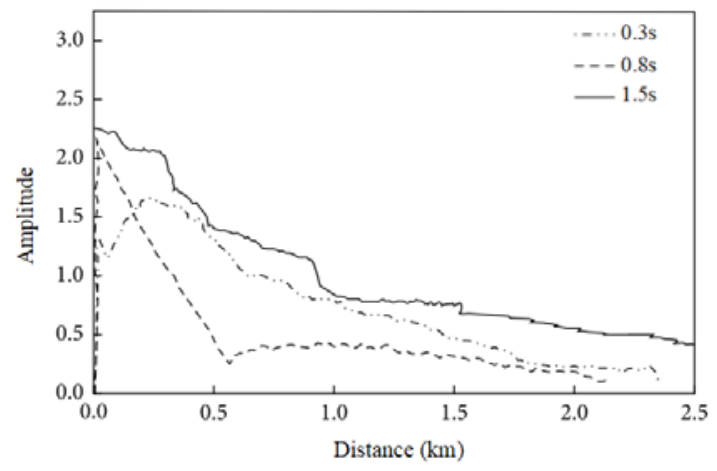

Figure 2 Normalized response curves of pulsed electric field in different time domain

The distribution of marine environmental noise is uncertain. Common noise distributions include Gaussian distribution and symmetric distribution, etc. However, in actual situations, the distribution of marine environmental noise is very complex. Higher-order statistics and their spectra can suppress Gaussian distribution and symmetric distribution noise. Fig. 3 is the result of superposition processing of measured ocean CSEM data for 20s and 100s. As can be seen from the figure, when the superposition window is 20s, compared with when the superposition window is 50 s, the signal amplitude is well preserved when the transceiving distance is small, but the noise level is obviously reduced when the transceiving distance is large, and an effective signal with a larger transceiving distance range can be obtained.

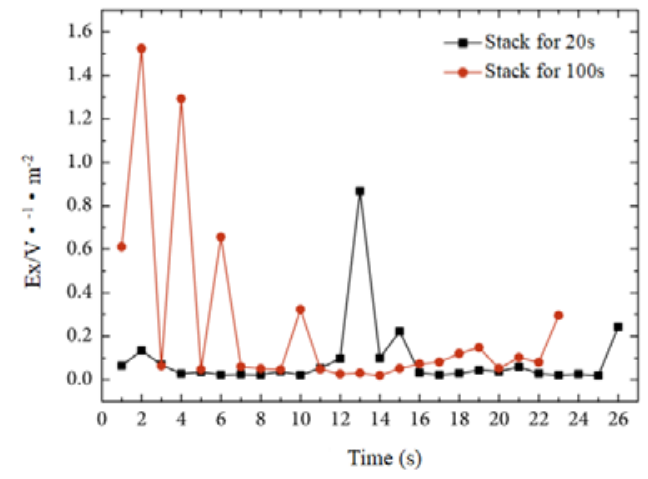

Figure 3 Comparison of superposition effect when the superposition window of measured ocean CSEM data is $20 \mathrm{~s}$ and $100 \mathrm{~s}$

Submarine electromagnetic acquisition stations record electromagnetic signals in time domain, which need to be converted into frequency domain signals by Fourier transform first. This paper uses slow Fourier transform to realize time-frequency conversion. Theoretically, the signal-to-noise ratio of the superimposed data is the product of the signal-to-noise ratio before superposition and the square root of the number of superimposed samples. However, the requirement of spatial sampling limits the amount of data that can be used for superposition. The frequency of the signal 
transmitted by the dipole source is a few known frequencies. This signal frequency can be considered as a useful signal of the active frequency, while other frequency signals are noise. In shallow sea environment, both frequency domain data and time domain data, with the increase of reservoir depth, the abnormal amplitude will increase. Coherent anisotropic component also increases gradually, which shows that the difference between total energy spectrum level and coherent anisotropic component spectrum level decreases. The amplitude of wave magnetic field will increase with the decrease of frequency, and also with the increase of wave height and velocity. In the investigation of wave spectrum, it is found that the frequency spectrum of wave induced magnetic field in geomagnetic field is a narrow band frequency spectrum. In order to improve the signal-to-noise ratio of seabed CSEM data, the most common method is to stack the data, but the requirements for ship speed and spatial sampling limit the amount of data that can be used for stacking. The law of resistivity change at different depths. These inversion methods and inversion processes are exactly the same as those of conventional magnetotelluric sounding data.

\section{Conclusion}

This paper presents a set of pretreatment methods for marine controllable source electromagnetic data. The processing of measured electromagnetic data from marine controllable sources shows that reliable results can be obtained by using slow Fourier transform. The basic performance parameters of working equipment for marine CSEM exploration are analyzed and introduced, including signal transmitter, submarine power supply dipole towing system and submarine multi-component receiving system. For oil and gas reservoirs in water depth of $200 \mathrm{~m}$, the data in frequency domain is enough to reflect the thickness and buried depth of the reservoir, thus increasing the joint inversion of time domain data without obvious improvement in resolution. The variation trend of the self-spectrum and cross-spectrum of marine environmental noise reaches a maximum at about $60 \mathrm{~Hz}$ at low frequency, and the spectrum level decreases with the increase of frequency and finally tends to be stable. The amplitude-frequency-time distribution diagram of time series is obtained through time-frequency directional spectrum analysis, which can well analyze the distribution of signal energy on the time-frequency plane.

\section{References}

[1] Du Jianli, Qin Guping, Li Zhenxing. Research on the application of big data technology in spectrum monitoring. China Radio, vol. 000, no. 010, pp. 52-53, 2018.

[2] Su Lixuan, Li Tianshi, Huo Bin. Application research of real-time spectrum analysis technology in electromagnetic radiation test and analysis of high-speed railway. China Railway, vol. 000, no. 007, pp. 70-75, 2019.

[3] Wu Tong, Li Diquan, Suo Guangyun, et al. Application of generalized S-transform timefrequency analysis in data processing of wide-area electromagnetic method. Geophysical and Geochemical Computing Technology, vol. 041, no. 003, pp. 379-385, 2019.

[4] Tian Yongpo, Zhang Gaojie. Application of Spectrum Analyzer in Electromagnetic Compatibility Test. Journal of Tongren University, vol. 019, no. 003, pp. 34-39, 2017.

[5] Zhu Zhongming, Zhang Shuangshi, Wang Kunpeng, et al. 2.5-dimensional forward modeling of frequency domain of electromagnetic method in marine oil and gas exploration. Science and Technology and Engineering, vol. 017, no. 011, pp. 152-156, 2017.

[6] Zhang Tianxin, Wang Zhongxing, Wu Shujun, et al. Research on ocean electromagnetic low clock drift and automatic gain acquisition technology. Chinese Journal of Geophysics, vol. 060, no. 011, pp. 4165-4173, 2017.

[7] Chen Guangyuan, Du Libin, Jing Jianen, et al. Research on 2.5-dimensional oceanic controlled source electromagnetic inversion algorithm and influence parameters. Progress in Geophysics, vol. 
no. 4, pp. 1796-1802, 2016.

[8] Cai Jianhua, Xiao Yongliang. Applicability analysis of Hilbert-Huang transform and Fourier transform in magnetotelluric data processing. Progress in Geophysics, vol. 032, no. 004, pp. 15481555, 2017. 